Documento No. 4

\title{
UNA APLICACIÓN DEL MODELO BAYESIANO DE DECISIÓN EN EL ANÁLISIS DE FUNCIONES DE PRODUCCIÓN AGRÍCOLAS
}

\author{
Por \\ Héctor E. González Méndez
}

Diciembre, 1978.

Versión Preliminar. No citar en publicación sin permiso del autor.

Las ideas contenidas en el presente ensayo son responsabilidad exclusiva del autor y no refleja la posición del Banco de México, S.A. 


\section{UNA APLICACIÓN DEL MODELO BAYESIANO DE DECISIÓN EN EL ANÁLISIS DE FUNCIONES DE PRODUCCIÓN AGRÍCOLAS ${ }^{*}$}

\section{HECTOR E. GONZALEZ MENDEZ}

A mediados del siglo pasado Von Liebig ${ }^{3}$ planteaba que los suelos que contenían todos los nutrientes necesarios para que creciera una planta, a excepción de uno, estaban vedados a todos aquellos productos agrícolas para los cuales ese nutriente faltante era indispensable.

Tal aseveración ha tenido muchas y variadas interpretaciones a través del tiempo, probablemente la más destaca es la que vincula al nombre del autor con la "Ley de lo mínimo". Esta ley postula que los rendimientos en la producción agrícola son proporcionales a la cantidad de nutrientes que el suelo posee, y que, cuando todos estos nutrientes se encuentran en las cantidades suficientes el aumentar la cantidad de uno o más no producirá ningún aumento en el rendimiento.

Los acontecimientos recientes, especialmente en el campo de la investigación sobre especies mejoradas y nuevas variedades, han venido a refutar acremente a la "Ley de lo mínimo". Sin embargo, cabe señalar que es con Von Liebig con quien se marca el inicio del estudio formal de las relaciones fundamentales entre los nutrientes del suelo y los rendimientos en la producción. Desde entonces a la fecha, ha sido preocupación de los científicos en suelos el conocimiento de la constitución funcional de las relaciones técnicas de producción.

Es el análisis y la discusión entre agrónomos sobre las funciones de producción, lo que atrae la atención del economista interesado en la cosa agrícola, y no a la inversa como en repetidas ocasiones se pretende. Sin embargo, la aportación del economista agrícola en la investigación e implementación de formas matemáticas explicativas de relaciones causales ha sido substancial. Inclusive, éste ha llegado

\footnotetext{
*Quiero manifestar mi agradecimiento al Doctor George W. Ladd, profesor de Economía de la Universidad del Estado de Iowa, por su interés en el análisis de la estadística bayesina aplicada.

${ }^{3}$ Liebig, Justus von. Die grundsätze der agricultur-chemie mit rücksicht anf die en England anestellten untersuchungen. Friedrich Viewing und Sohn. Braunschweig 1855. (Cita de Earl O. Heady, John L. Dillon. Agricultural Production Functions, Iowa State University Press, Ames, Iowa 1972, p. 10).
} 
a un nivel de complejidad en el análisis teórico que en repetidas ocasiones parece divorciarse por completo de la realidad.

A partir de Knut Wicksell ${ }^{4}$ (quien fue posiblemente el primer economista que surgiere una forma algebraica para las funciones de producción agrícola) el análisis de funciones de producción continuas (por tanto diferenciables) para fenómenos físicos en la agricultura aumenta rápidamente su incidencia en las revistas especializadas. La razón del interés en estimar funciones de producción agrícolas se debe en gran parte a que éstas proveen conocimiento científico básico en la explicación de un todo más amplio. Cabe mencionar, sin embargo, que a medida que este interés crece entre los economistas, los agrónomos comienzan a manifestar en forma también creciente su insatisfacción hacia las expresiones algebraicas puras que tienden a divorciar el fenómeno causal puro, de interés para el economista, de la realidad del medio natural en que el fenómeno se presenta. La agricultura se encuentra rodeada por condiciones de incertidumbre, las funciones de producción continuas sucumben ante tal realidad, inclusive aquellas estimaciones que contemplan márgenes de error para cada punto de la curva de insumo producto.

La predicción de un nivel de producción, resultado de un nivel determinado de insumos, y la toma de decisiones basada en esta predicción son dos de los problemas más complejos encarados por la planeación agrícola. Existe una gran cantidad de investigación económica sobre la toma de decisiones en condiciones de incertidumbre, sin embargo, los modelos teóricos destinados a estimar funciones de producción se encuentran aún ajenos a los avances recientes en este campo teórico. El presente ejercicio tiene por objeto incorporar a nivel teórico un modelo bayesiano para la toma de decisiones en el uso de funciones de producción agrícolas.

\section{La Función de Producción}

La teoría de la producción descansa indiscutiblemente sobre consideraciones de tipo tecnológico. Se contiene información acerca de un determinado número de insumos susceptibles de ser transformados en un producto determinado, la cuestión es saber en que proporción dicha transformación es factible. La respuesta depende del avance tecnológico o estado actual de la tecnología asequible. La función de producción es una relación técnica que nos dice cual es la cantidad

${ }^{4}$ Wicksell Knut. Den "Kritska punkten" i lagen för Jordbrukets altagande produktivitet. Ekinomisk Tidskrift. 1916 pp. 285-292. (Ibid., p. 15) 
máxima de producto capaz de ser producida por todos y cada uno de los conjuntos especificados de insumos (o factores de la producción) y esta relación se define dado el estado actual del conocimiento tecnológico ${ }^{3}$

La función de producción como una relación física entre un insumo y un producto puede ser gráficamente representada como en la figura 1. Existen muchas y muy variadas posibilidades algebraicas y gráficas para representar una función de Producción, la que aquí aparece es tal vez la más popular para fines académicos; la curva contiene una primera parte con rendimientos crecientes de la escala (donde el producto aumenta más que proporcionalmente respecto a los aumentos en el insumo) le sigue una segunda fase de rendimientos decrecientes de la escala (donde el producto aumenta menos que proporcionalmente a los aumentos del insumo) y por último una fase de rendimientos totales decrecientes.

Es claro que cualquier punto de la función continua de producción puede ser interpretada para fines prácticos como una predicción, ni perfecta, ni tampoco totalmente imperfecta, sobre un evento esperado. Las condiciones climáticas, fundamentalmente, evitan que la relación causal se presente en forma perfecta. Sin embargo, siempre es loable definir un rango dentro del cual la probabilidad de que se localice el nivel de producción para un nivel dedo de insumo es máxima. Este rango de acción de la relación causal puede ser tan amplio como se desee, puede ir de una frontera inferior igual a cero (Producción igual a cero) hasta una frontera superior que describa una relación singular insumo producto sólo observaba en condiciones excepcionales de laboratorio. La práctica cotidiana entre aquéllos que viven del producto del campo impone límites tanto al optimismo como el pesimismo; ubicando a la frontera inferior de factibilidad de la función de producción por encima de cero, (aunque cero-producto es siempre un evento plausible) y a la frontera superior a un nivel alto alguna vez observado. Para fines prácticos, la frontera superior se encuentra más íntimamente ligada a los puntos definidos por la función técnica de producción, esto es, en la mayoría de los casos los resultados de la relación continua entre insumo y producto de un centro agrícola de investigación es vista por el agricultor como la frontera superior misma. Las condiciones bajo las cuales el agrónomo determina los puntos de la relación entre niveles de insumo y sus consecuentes niveles de insumo y sus consecuentes niveles de productos son, en términos generales, las óptimas; de tal manera que la relación estimada por

\footnotetext{
${ }^{3}$ Samulson, Paul A. Economics Ninth Edition, Inc. Graw Hill p. 535.
} 
la regresión de los niveles de producción, es vista como un nivel "ideal" de rendimiento para el agricultor común que se enfrenta al medio en condiciones normales.

\section{Figura 1}

\section{La Función de Producción}

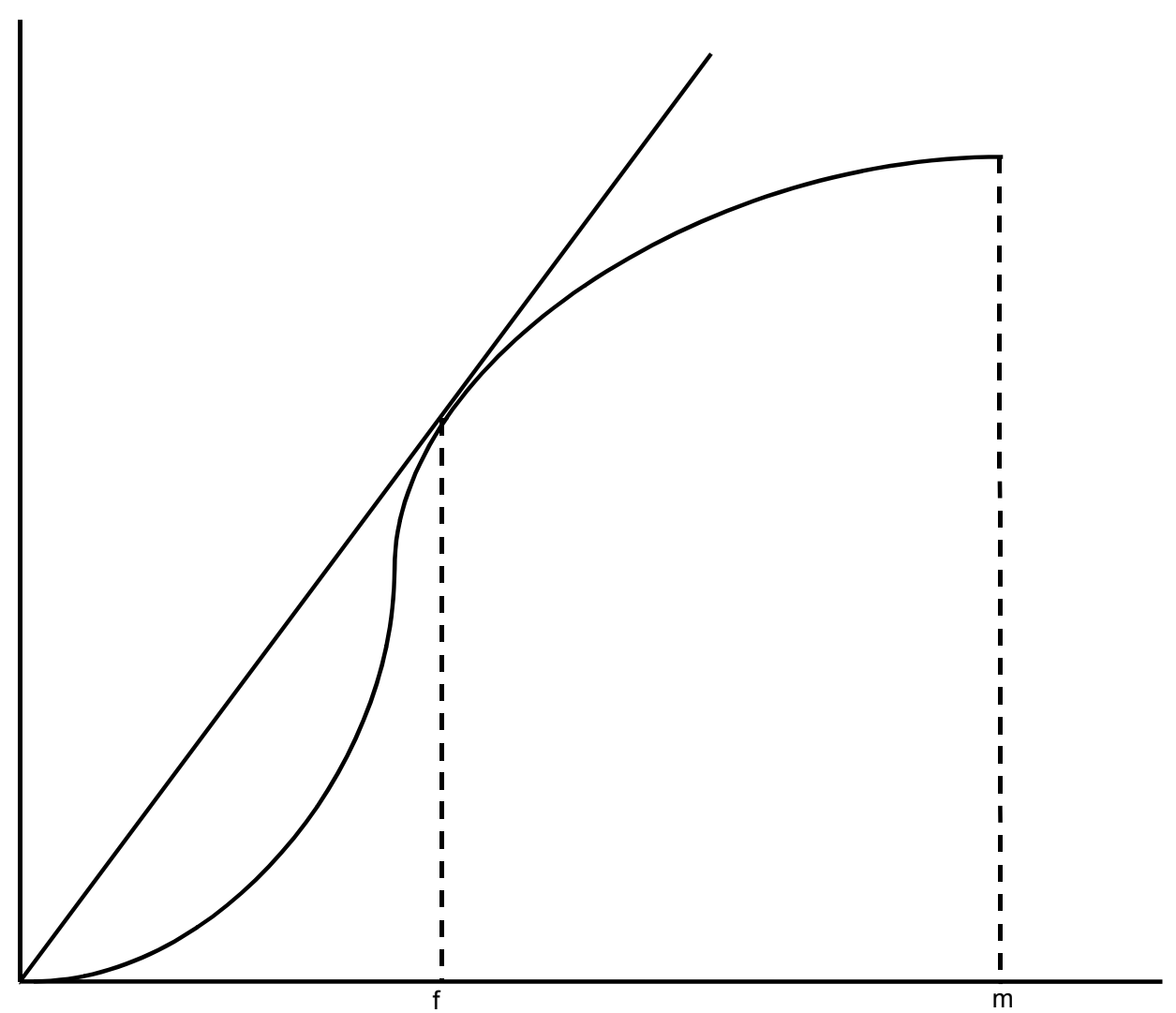

\section{El rango de factibilidad empírica de la función teórica de Producción}

Proponemos que la incidencia de la relación causal insumo-producto empírica puede mantener una relación probabilística con la función teórica de Producción del tipo de la distribución de Poisson. Por lo tanto, los puntos de la curva de la función de producción representan las medidas de las distribuciones correspondientes a todos los valores posibles de X (el insumo). Bajo este supuesto, la relación que nosotros tratamos de definir es como sigue:

$$
q=f(X) ; \text { la función de Producción }
$$


Donde los valores q de la función de producción para cada nivel de insumo representan el valor esperado (medio) de la distribución de Poisson, esto es:

$$
\mathrm{E}(\mathrm{q} / \mathrm{X})=\mu(\mathrm{q} / \mathrm{X})
$$

y

$$
P(q / X)=\frac{e^{-\mu} \mu q}{q !}
$$

El rango de factibilidad empírica de la función teórica de producción se define por el área bajo la curva de probabilidad a un nivel de confianza de 95.0\%. La figura 2 es una representación hipotética de lo que aquí se argumenta.

\section{Figura 2}

Rango de factibilidad empírica de una función teórica de producción ${ }^{4}$

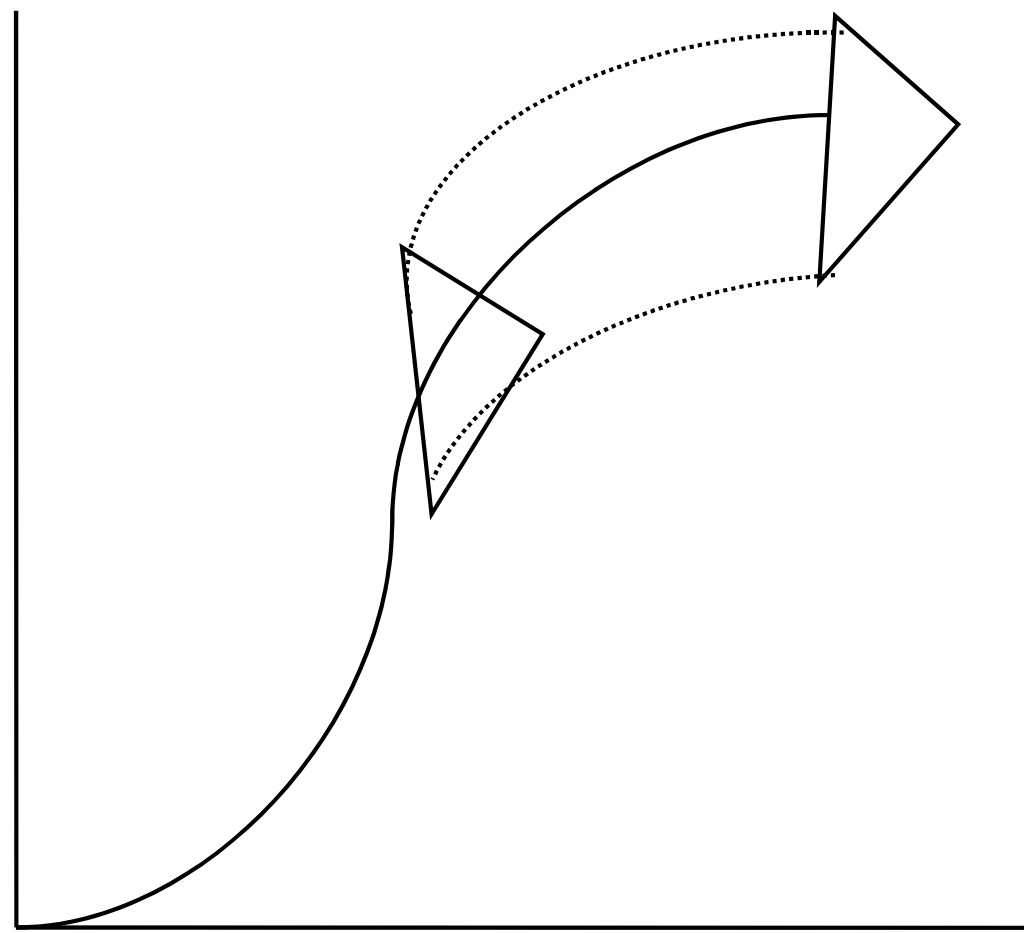

\footnotetext{
${ }^{4}$ La representación gráfica no corresponde en rigor a la distribución de Poisson, ya que esta es discreta. Sin embargo, resulta conveniente a los fines que se persiguen.
} 
En la figura 2 la función teórica de producción es la línea continua, mientras que las líneas punteadas representan el rango de factibilidad empírica de la producción.

Resta por analizar el comportamiento de la producción dentro del rango de factibilidad empírica tomando en consideración tanto la experiencia del agricultor en el campo de lo práctico, como la información estadística sobre producción en ciclos agrícolas anteriores o en lugares distintos con características similares. El análisis Bayesiano de probabilidad parece proveer los medios necesarios para determinar el nivel esperado de producción tomando en cuenta tanto la información histórica disponible sobre rendimientos observados en la práctica; como la información que se desprende de encuestas de opinión sobre rendimientos entre agricultores o especialistas en la materia. Es la posibilidad de la estadística bayesiana de combinar información teórica con información empírica la que la hace un instrumento ideal en la implementación de las funciones de producción. El concepto de incertidumbre toma un significado completamente diferente cuando uno pasa de la estadística clásica a la estadística bayesiana. La incertidumbre ha sido general utilizada para describir una situación en la cual el resultado de una acción determinada no es perfectamente predecible debido a la existencia ya sea de parámetros cuyos valores son desconocidos o debido a un término (siempre presente) aleatorio de error. El método tradicional para analizar la incertidumbre ha sido el suponer una distribución probabilística para el término de error, mas no para los valores desconocidos de los parámetros. En el método bayesiano, todos los elementos sujetos a incertidumbre pueden ser representados por distribuciones probabilísticas. Consideramos que con la ayuda de estadística bayesiana es posible tabular, previo a los trabajos de siembra, todos los niveles de producción esperados que reflejen aspectos objetivos y subjetivos, técnicos y empíricos propios de una situación agrícola específica.

\section{EL METODO BAYESIANO PARA LA TOMA DE DECISIONES *}

Las características distintivas de la estadística bayesiana son:

a) La interpretación personal de la probabilidad.- Es legítimo cuantificar "apreciaciones" sobre incertidumbre en términos numéricos subjetivamente determinados.

\footnotetext{
* El análisis de esta sección está basada en el trabajo de P.E. Green y D.S. Tull. "Research for marketing decision". Englenwood Cliffs, New Jersey Prentice Hall Inc. 1966.
} 
b) El uso de datos de series cronológicas como sistema rector de expectativas; es factible incorporar en el análisis de predicción las experiencias acumuladas en series cronológicas.\}

Existen un sin número de elementos en la Teoría Bayesiana, sin embargo nosotros nos hemos de concentrar sólo en aquéllos que son indispensables a nuestro problema y que son:

1) La función objetivo del análisis.- Se tratará de maximizar el rendimiento esperado por unidad de insumo.

2) El marco en que se desarrollo el Problema.- Se trata de identificar el campo de acción de la variable dependiente, en nuestro caso ésta se limita al rango de factibilidad empírica para cada nivel de insumo de la función de producción.

3) Cursos alternativos de acción. Un curso de acción es la especificación de una medida a tomar que es factible. En nuestro caso los cursos de acción serán los distintos niveles de insumo aplicados en el proceso de producción dentro del área económica de la producción, comúnmente conocida como la fase II en la Teoría de la Producción ${ }^{3}$.

4) Método de Selección de la acción. La solución del problema está en seleccionar el mejor curso de acción congruente con el objetivo buscado. El marco en el que se desarrolla el problema asume que se tiene un conocimiento limitado del resultado de una acción, esto es, para cada nivel de insumo existe una gama de posibles niveles de producto, a los cuales se les adjudica una probabilidad determinada. Es aquí donde hemos de introducir tres elementos de probabilidades, los cuales permiten determinar el nivel esperado de producción.

a. La distribución de Probabilidad apriorística. Esta distribución de Probabilidad apriorística. Esta distribución se desprende del juicio apriorístico sobre las condiciones actuales para el ciclo de producción sobre le cual se está por decidir el nivel del insumo. Llamamos $\mathrm{P}\left(\theta_{\mathrm{ji}}\right)$ a la probabilidad apriorística del nivel de producción $\mathrm{j}$ vinculado a el nivel de insumo i. Estos es, existe una probabilidad apriorística para cada nivel de producción dentro del rango de factibilidad empírica de la función teórica de producción y para cada nivel de insumo o curso de acción.

\footnotetext{
${ }^{3}$ C.E. Ferguson, Microeconomic Theory; Third Edition, Richard D. Irwin, Inc. Homewood, Illinois. Page 150
} 
b. La distribución de Probabilidad Condicional. Esta distribución está basada en el análisis de datos, es la frecuencia relativa que se desprende de comparar predicciones y resultados sobre rendimiento en procesos similares o de años anteriores. Esto es, la frecuencia relativa con que la predicción $Z_{k}$ (cuando $k$ es un nivel determinado de producción) se observó cuando $\theta_{\mathrm{j}}$ ocurrió al nivel de insumo i se define algebraicamente como $\mathrm{P}\left(Z_{\mathrm{k}} / \theta_{\mathrm{j}}\right)_{\mathrm{i}}$ y se denomina como la probabilidad condicional del evento $Z_{\mathrm{k}}$ dado el evento $\theta_{\mathrm{j}}$.

c. La distribución de Probabilidad Posterior. Esta distribución es el resultado de combinar toda la información apriorística con toda la información condicional. La probabilidad posterior se define algebraicamente como; $\mathrm{P}\left(\theta_{\mathrm{j}} / \mathrm{Z}_{\mathrm{k}}\right)_{\mathrm{i}} \mathrm{y}$ nos indica la probabilidad de observar el nivel de producción $\theta_{\mathrm{j}}$ en el i-ésimo nivel de insumo, dado que se conoce la predicción $Z_{k}$ acerca del nivel esperado para el ciclo agrícola en cuestión. La probabilidad posterior $\mathrm{P}\left(\theta_{\mathrm{j}} / \mathrm{Z}_{\mathrm{k}}\right)_{\mathrm{i}}$ se obtiene mediante el uso del teorema de Bayes como se presenta más adelante.

La única restricción que se impone al modelo es que las probabilidades apriorística, condicional y posterior no sean negativas y sumen uno (1.0) en cada caso.

\section{DESARROLLO DEL MODELO}

Supongamos una función teórica de producción, digamos entre el uso de fertilizantes y los rendimientos por hectárea de maíz, la cual puede quedar debidamente representada por la ecuación (1). La aplicación del insumo se cuenta en unidades discretas que van de cero a infinito. Sin embargo, nosotros solamente estamos interesados por aquellos niveles de insumo ubicados dentro de la región económica de la función de producción. Esto significa que los cursos de acción $\left(\mathrm{a}_{\mathrm{i}}\right)$ de que dispone el agricultor corresponden a los volúmenes crecientes de insumo aplicables (kilogramos por ejemplo) y que van de un volumen de producción donde el producto promedio alcanza un máximo al nivel de producción donde el producto marginal se iguala a cero (de f a m en la figura 1.)

Estados de la Naturaleza.- Mencionamos anteriormente que el resultado final de una acción determinada depende en gran medida de eventos cuya ocurrencia está fuera de control de quien toma la acción. La presencia o ausencia de un sin de un sin número de eventos aleatorios de este tipo en la 
producción agrícola nos conducen a considerar un conjunto de niveles de producción para cada nivel de insumo. A estos niveles de producción probables se les llama "Estados de la Naturaleza" y el grado de conceptualización acerca de ellos es crucial a la solución del problema de decisión. El intervalo de la distribución de Poisson que engloba 95.0\% de los eventos (niveles de producción) probables se fracciona en $\mathrm{n}$ intervalos discretos; todos igualmente probables, mutuamente excluyentes $\mathrm{y}$ colectivamente exhaustivos de la distribución de probabilidad de Poisson comprendida entre las fronteras superiores e inferior del rango de factibilidad empírica. Cada fracción resultante es un "Estado de la Naturaleza" o evento igualmente probable, denominado algebraicamente como $\theta_{\mathrm{ji}}$ donde $\mathrm{i}=\mathrm{f}, \mathrm{f}+1, \mathrm{f}+2, \ldots, \mathrm{m}$ y $\mathrm{j}=1,2, \ldots \mathrm{n}$. En otras palabras $\theta_{\mathrm{ji}}$ es el j-ésimo estado de la naturaleza para el $\mathrm{i}$ ésimo nivel de insumo.

$$
P(\theta)=\sum_{j=1}^{n} P\left(\theta_{j i}\right)=.95 V_{i}
$$

Para $\quad P(\theta)=P\left(q_{a i} \geq q_{i} \geq q_{b i}\right)$

$$
\mathrm{y} \quad \mathrm{P}\left(\theta_{\mathrm{ji}}\right)=\frac{.95}{\mathrm{n}} \mathrm{V}_{\mathrm{j}}
$$

donde $\mathrm{q}_{\mathrm{ai}} \mathrm{y} \mathrm{q}_{\mathrm{bi}}$ son las fronteras superior e inferior respectivamente del rango de factibilidad empírica de $\mathrm{X}_{\mathrm{i}}$. Los valores fuera de este rango son improbables estadísticamente hablando y por tanto se excluye de los estado de la naturaleza que considera la producción.

Matriz de Producto.- La decisión del agricultor por optar por un nivel $\mathrm{X}_{\mathrm{i}}$ de insumo se llamó acción $\mathrm{a}_{\mathrm{i}}$. Se asumió que el agricultor conoce a ciencia cierta los niveles de insumo entre los cuales puede optar, que van de k a m. Por otra parte, se identificó el rango de factibilidad de los estados de la naturaleza para todos y cada uno de los niveles de insumo entre los cuales el agricultor puede optar.

Para cada par (acción) - (estado de la naturaleza) es necesario identificar el consecuente nivel de producto. El valor medio de cada intervalo de probabilidad o "estado de la naturaleza" se define como el nivel de producto esperado $\mathrm{q}_{\mathrm{ij}}$ ' esto es, el producto del j-ésimo estado de la naturaleza, dado el i-ésimo nivel de insumo empleado. Los "productos esperados" de todas las posibles combinaciones entre acciones $a_{i} y$ estados de la naturaleza $\theta_{j}$ proporcionan un conjunto de posibilidades mutuamente 
excluyentes y colectivamente exhaustivas. El conjunto así definido se llama "Matriz de Producto". Los elementos de la Matriz son niveles de producción esperados. La tabla 1 presenta un ejemplo de la Matriz de Producto.

Tabla 1. Matriz de Producto

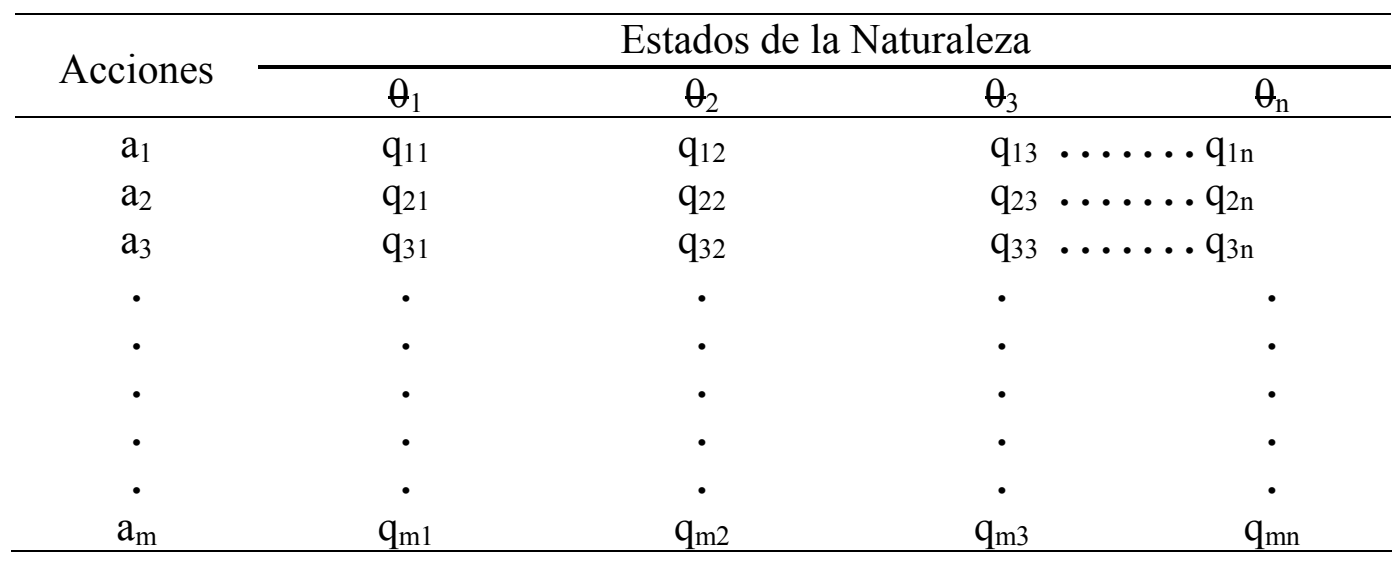

\section{$\underline{\text { PROBABILIDADES APRIORISTICAS }}$}

La importancia que se de a la selección de probabilidades apriorísticas $\mathrm{P}_{\mathrm{a}}\left(\theta_{\mathrm{ji}}\right)$ es crucial a la validez de los resultados. La información apriorística puede originarse de la observación casual o de la introspección de algunas consideraciones teóricas del agricultor. Esto es, el agricultor como unidad de decisión hace uso de su información apriorística (basada en su experiencia y en la de expertos y agencias agrícolas a su alcance) a fin de atribuir probabilidades a los niveles de producción de la matriz de producto.

La función de densidad de la probabilidad apriorística se convierte en la distribución de probabilidades asignadas por el agricultor a cada estado de la naturaleza para cada nivel de insumo. Resulta importante el hacer notar que la distribución apriorística de un agricultor puede perfectamente diferir de la de otro, aunque ambos confronten circunstancias idénticas; de aquí se desprende el carácter personalizado de las predicciones.

\section{PROBABILIDAD CONDICIONAL}

En la mayoría de los casos las zonas de producción agrícola disponen de servicios meteorológicos con pronósticos de lluvias, humedad, plagas, etc., al principio de cada ciclo agrícola. 
Estos pronósticos climatológicos se encuentran íntimamente relacionados con los niveles de producción asequibles para cada nivel de insumo. En primer término es necesario identificar el pronóstico climatológico emitido al inicio del ciclo agrícola con los estados de la naturaleza, por ejemplo un pronóstico de sequía y plaga conllevaría a un pronóstico de producción muy bajo. El problema de la probabilidad condicional es el de encontrar la incidencia de los pronósticos de producción sobre los estados de la naturaleza, esto es, sobre una muestra de ciclos agrícolas se pretende determinar la frecuencia relativa de pronósticos de estados de la naturaleza sobre estados de la naturaleza que ocurrieron en el pasado o en zonas agrícolas semejantes a la estudiada. En otras palabras, una comparación entre predicciones y sucesos nos proporciona una frecuencia relativa del pronóstico sobre su campo de acción. Por tanto, la probabilidad condicional $P_{c}\left(Z_{k} / \theta_{j}\right)_{i}$ se define como la fracción de veces que la predicción $Z_{k}(k=1,2, \ldots n)$ ocurrió en una muestra de ciclos agrícolas en los que se observaron estados de la naturaleza $\theta_{j}(j=1, \ldots n)$. Si $P_{c}\left(Z_{k} / \theta_{j}\right)$ i es aproximadamente cero cuando $\mathrm{k}=\mathrm{j}$ y la muestra de ciclos agrícolas es de tamaño adecuado, se debe concluir que los pronósticos $Z_{\mathrm{k}}$ (acerca de los estados de la naturaleza) no son en efecto buenas predicciones de los eventos $\theta_{\mathrm{j}}$; en estos casos la distribución de probabilidades apriorísticas se convierte en la información probabilística más valiosa

\section{$\underline{\text { PROBABILIDAD POSTERIOR }}$}

La probabilidad posterior es el resultado de combinar la probabilidad apriorística con la probabilidad condicional a través del Teorema de Bayes, esto es, la probabilidad posterior depende de las probabilidades apriorística y condicional exclusivamente y se le define como $\mathrm{P}_{\mathrm{p}}\left(\mathrm{Z}_{\mathrm{k}} / \theta_{\mathrm{j}}\right)_{\mathrm{i}}$ que se interpreta como la probabilidad de que ocurra el estado de la naturaleza $\theta_{\mathrm{j}}$ dado que existe una predicción $Z_{\mathrm{k}}$ para el i-ésimo nivel de insumo. La función de densidad de la probabilidad posterior se calcula por la ecuación de Bayes que sigue:

$$
\mathrm{p}_{\mathrm{p}}\left(\theta_{\mathrm{j}} / Z_{\mathrm{k}}\right)_{\mathrm{i}}=\frac{\operatorname{Pa}(\theta \mathrm{ji}) \operatorname{Pc}(Z \mathrm{k} / \theta \mathrm{j}) \mathrm{i}}{\operatorname{Pm}(Z \mathrm{ki})} \quad \mathrm{i}=1, \ldots \mathrm{m}
$$

$\mathrm{P}_{\mathrm{m}}\left(Z_{\mathrm{ki}}\right)$ es la función de densidad de la probabilidad marginal de observar la k-ésima predicción del rango de factibilidad empírica $Z(Z=\theta)$ para el i-ésimo nivel de insumo y se obtiene como sigue: 


$$
\mathrm{p}_{\mathrm{m}}\left(\mathrm{Z}_{\mathrm{ki}}\right)=\sum_{\mathrm{j}=1}^{\mathrm{n}} \mathrm{P}_{\mathrm{a}}\left(\theta_{\mathrm{ji}}\right) \mathrm{P}_{\mathrm{c}}\left(\mathrm{Z}_{\mathrm{k}} / \theta_{\mathrm{j}}\right)_{\mathrm{i}} \quad \mathrm{i}=1, \ldots \mathrm{m}
$$

La función de probabilidad posterior sirve para hacer predicciones acerca de los valores de producción esperados (estados de la naturaleza) en el ciclo agrícola próximo tomando en consideración el punto de vista del agricultor acerca del porvenir (basado en su experiencia) y del comportamiento pasado de predicciones de producción (basadas en pronósticos de tiempo) respecto a su incidencia con la realidad observada.

Existen varias formas de ordenar los elementos discretos de probabilidad de la distribución de probabilidades posteriores $\mathrm{P}_{\mathrm{p}}\left(\theta_{\mathrm{j}} / \mathrm{Z}_{\mathrm{k}}\right)_{\mathrm{i}}$. Nosotros hemos de optar por mantener $\mathrm{k}$ constante, dejando variar i y j. En otras palabras, $\mathrm{P}_{\mathrm{p}}\left(\theta_{\mathrm{j}} / Z_{\mathrm{k}=\text { const. }}\right)_{\mathrm{i}}$ combina sólo un intervalo de predicción $\mathrm{k}$ con todos los estados de la naturaleza comprendidos por el rango de factibilidad empírica y todos los niveles de insumo de $\mathrm{f} \mathrm{a} \mathrm{m}$. A este tipo de arreglos les llamamos "Matrices de Predicciones Posteriores de Probabilidad", esto es, 1P 1P 1P ${ }_{k}$. La tabla siguiente presenta un ejemplo de estas matrices.

Tabla 2. Matriz de Predicciones Posteriores de Probabilidad $\mathrm{P}_{\mathrm{p}}\left(\theta_{\mathrm{j}} / \mathrm{Z}_{\mathrm{k}=\text { const. }}\right)_{\mathrm{i}}$

\begin{tabular}{|c|c|c|c|}
\hline \multirow{2}{*}{$\begin{array}{c}\text { Estado de la } \\
\text { naturaleza }\end{array}$} & \multicolumn{3}{|c|}{ NIVELES DE INSUMO } \\
\hline & $X_{f}$ & $X_{f}$ & $X_{\mathrm{m}}$ \\
\hline$\theta_{1}$ & $\mathrm{P}_{\mathrm{p}}\left(\theta_{1} / \mathrm{Zk}\right)_{1}$ & $\mathrm{P}_{\mathrm{p}}(\theta$ & . $\mathrm{P}_{\mathrm{p}}\left(\theta_{1} / \mathrm{Z}_{\mathrm{k}}\right)_{\mathrm{m}}$ \\
\hline$\theta_{2}$ & $\mathrm{P}_{\mathrm{p}}\left(\theta_{2} / \mathrm{Zk}\right)_{1}$ & $\mathrm{P}_{\mathrm{p}}(\theta$ & $\mathrm{P}_{\mathrm{p}}\left(\theta_{2} / \mathrm{Z}_{\mathrm{k}}\right)_{\mathrm{m}}$ \\
\hline$\theta_{3}$ & $\mathrm{P}_{\mathrm{p}}\left(\theta_{3} / \mathrm{Z}_{\mathrm{k}}\right)_{1}$ & & $P_{p}\left(\theta_{3} / Z_{k}\right)_{m}$ \\
\hline - & - & - & - \\
\hline - & - & - & - \\
\hline$\dot{\theta}_{\mathrm{n}}$ & $\mathrm{P}_{\mathrm{p}}\left(\dot{\theta_{\mathrm{n}}} / \mathrm{Z}_{\mathrm{k}}\right)_{1}$ & $\mathrm{P}_{\mathrm{p}}(\theta$ & $\mathrm{P}_{\mathrm{p}}\left(\theta_{\mathrm{n}} / \dot{Z}_{\mathrm{k}}\right)_{\mathrm{m}}$ \\
\hline
\end{tabular}

\section{ESTRATEGIAS BAYESIANAS}

Una estrategia Bayesiana es la selección de un curso específico de acción el cual maximiza la medida ponderada de los productos de la matriz de producto. Las ponderaciones no son otra cosa que una distribución determinada de probabilidad sobre los estados de la naturaleza. Existen dos tipos de estrategias bayesianas que son "SIN DATOS" y "CON-DATOS", las cuales dependen de las probabilidades apriorística y posterior respectivamente. 
1.- Bajo el método conocido como "SIN-DATOS" el producto esperado de la acción $a_{i}$ es qexp $\left(a_{i}\right)$ y que se define como sigue:

$$
\mathrm{q}_{\exp }\left(\mathrm{a}_{\mathrm{i}} / \mathrm{Z}_{\mathrm{k}}\right)=\sum_{\mathrm{j}=1}^{\mathrm{n}} \mathrm{q}_{\mathrm{ij}} \cdot \mathrm{P}_{\mathrm{a}}\left(\mathrm{O}_{\mathrm{ji}}\right) \quad \mathrm{i}=\mathrm{f}, \ldots \mathrm{m}
$$

La estrategia bayesiana "SIN-DATOS" consiste en seleccionar el curso de acción $\mathrm{a}_{\mathrm{i}}$ " tal que el producto esperado $\mathrm{q}_{\exp }\left(\mathrm{a}_{\mathrm{i}}\right)$ sea el máximo, esto es:

$$
\mathrm{q}_{\exp }\left(\mathrm{a}_{\mathrm{i}}^{*}\right)=\max _{\mathrm{i}} \mathrm{q}_{\exp }\left(\mathrm{a}_{\mathrm{i}}\right) \quad \mathrm{i}=\mathrm{f}, \ldots, \mathrm{m}
$$

2.- Bajo el método conocido como "CON-DATOS" el producto esperado de la acción $a_{i}$ dada una función posterior de probabilidad y una predicción $Z_{k}$ es $q_{\exp }\left(\mathrm{a}_{\mathrm{i}} / Z_{\mathrm{k}}\right)$ y se computa como sigue:

$$
\begin{gathered}
\mathrm{q}_{\exp }\left(\mathrm{a}_{\mathrm{i}} / Z_{\mathrm{k}}\right) \sum_{\mathrm{j}=1}^{\mathrm{n}} \mathrm{q}_{\mathrm{ij}} \cdot \mathrm{P}_{\mathrm{p}}\left(\theta_{\mathrm{i}} / Z_{\mathrm{k}}\right)_{\mathrm{i}} \quad \mathrm{i}=\mathrm{f}, \ldots \mathrm{m} \\
\mathrm{k}=1, \ldots \mathrm{n}
\end{gathered}
$$

La estrategia bayesiana "CON-DATOS", una vez conocidas las predicciones para el ciclo agrícola venidero, consiste en seleccionar el curso de acción $\mathrm{a}_{\mathrm{i}}{ }^{*} \mathrm{Z}_{\mathrm{k}}^{*}$ (donde $\mathrm{Z}_{\mathrm{k}}$ * es la predicción particular para el ciclo en cuestión). En cada caso, habrá $\underline{\mathrm{n}} \mathrm{Z}_{\mathrm{k}}{ }^{\prime} \mathrm{s}$ por nivel de insumo $\mathrm{x}$, sin embargo, solamente habrá un $Z_{\mathrm{k}}$ por nivel de insumo y por ciclo agrícola. Por tanto, existe sólo un $\mathrm{a}_{\mathrm{i}} / \mathrm{Z}_{\mathrm{k}} *$ por $\mathrm{Z}_{\mathrm{k}}$ y sólo un $\mathrm{a}_{\mathrm{i}}{ }^{* *} / \mathrm{Z}_{\mathrm{k}} *$ por función de producción. El curso de acción seleccionado debe de maximizar el producto esperado $\mathrm{q}_{\mathrm{exp}}\left(\mathrm{a}_{\mathrm{i}} / \mathrm{Z}_{\mathrm{k}^{*}}\right)$ como sigue:

$$
\left.\mathrm{q}_{\exp }\left(\mathrm{a}_{\mathrm{i}}^{* *}\right) / \mathrm{Z}_{\mathrm{k}}\right)=\max _{\mathrm{i}}\left(\mathrm{a}_{\mathrm{i}}^{*} / \mathrm{Z}_{\mathrm{k}^{*}}\right) \quad \mathrm{i}=1, \ldots \mathrm{m}
$$

donde las $\mathrm{k}^{*}$ 's son los intervalos relevantes respecto al conjunto de predicciones $Z_{\mathrm{k}}$.

Para ambas estrategias (CON-DATOS o SIN-DATOS) el resultado es una modificación de la función continua de producción en una sucesión de valores esperados discretos de producción, entre los 
cuales sólo uno maximiza el rendimiento (esperado) por unidad de insumo. En la figura 3 se presenta hipotéticamente lo que hasta aquí se ha argumentado.

Figura 3. La función teórica de producción y la función empírica bayesiana.

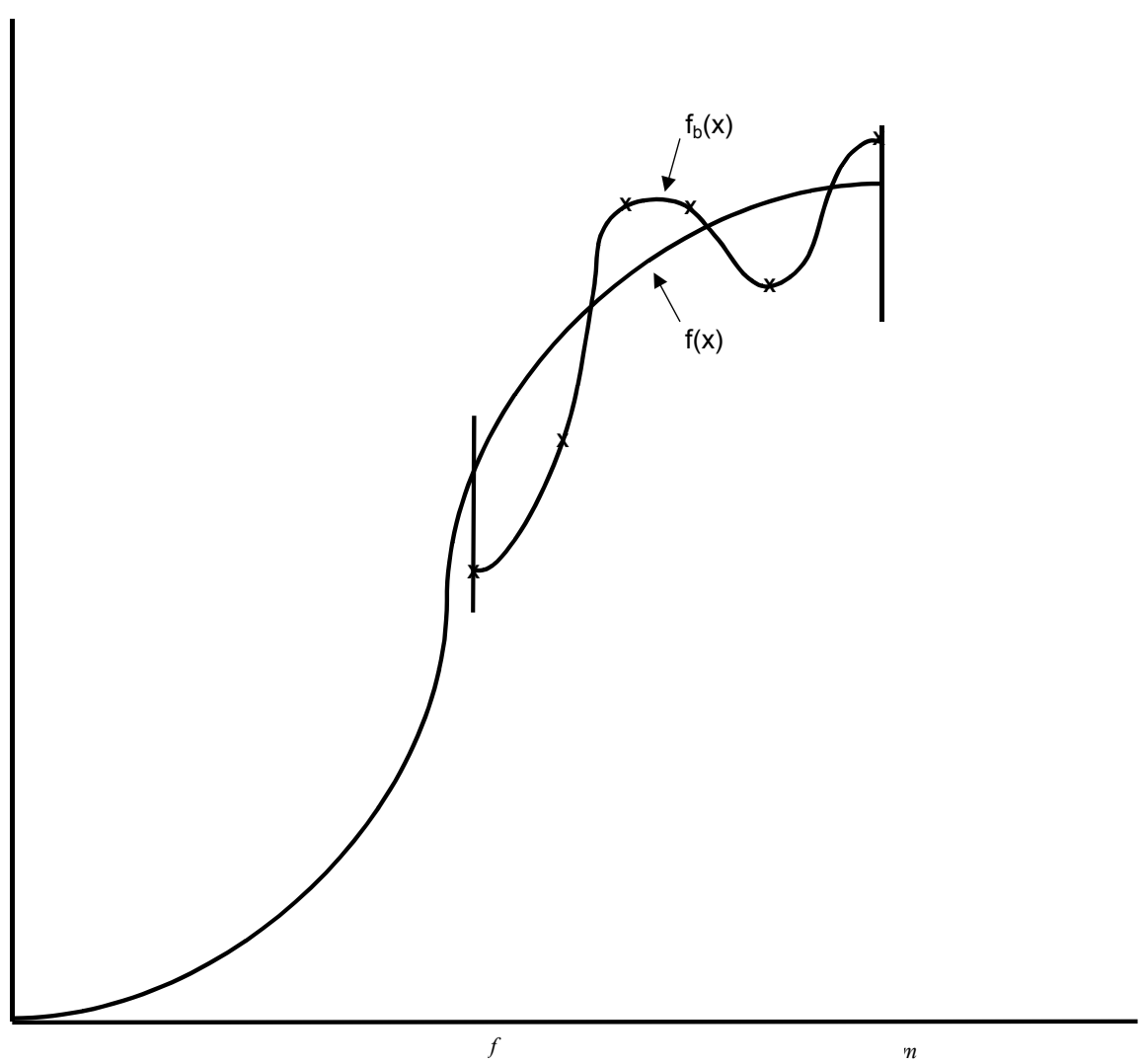

